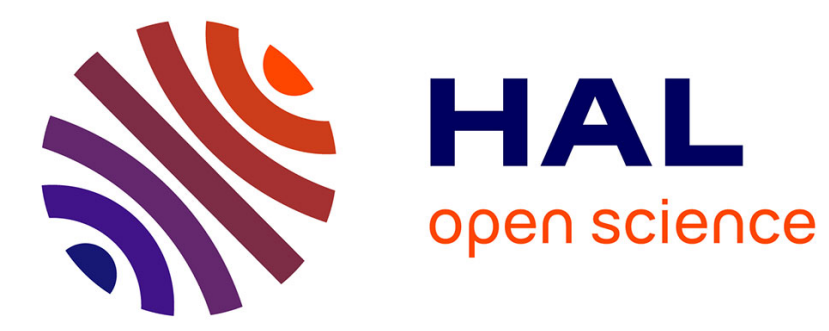

\title{
Cerebral vascular atlas generation for anatomical knowledge modeling and segmentation purpose
}

Nicolas Passat, Christian Ronse, Joseph Baruthio, Jean-Paul Armspach, Claude Maillot

\section{> To cite this version:}

Nicolas Passat, Christian Ronse, Joseph Baruthio, Jean-Paul Armspach, Claude Maillot. Cerebral vascular atlas generation for anatomical knowledge modeling and segmentation purpose. Computer Vision and Pattern Recognition (CVPR), 2005, San Diego, United States. pp.331-337, 10.1109/CVPR.2005.97 . hal-01694969

\section{HAL Id: hal-01694969 \\ https://hal.univ-reims.fr/hal-01694969}

Submitted on 26 Feb 2018

HAL is a multi-disciplinary open access archive for the deposit and dissemination of scientific research documents, whether they are published or not. The documents may come from teaching and research institutions in France or abroad, or from public or private research centers.
L'archive ouverte pluridisciplinaire HAL, est destinée au dépôt et à la diffusion de documents scientifiques de niveau recherche, publiés ou non, émanant des établissements d'enseignement et de recherche français ou étrangers, des laboratoires publics ou privés. 


\section{Cerebral Vascular Atlas Generation for Anatomical Knowledge Modeling and Segmentation Purpose}

\author{
N. Passat, C. Ronse \\ LSIIT, UMR 7005 CNRS-ULP \\ Strasbourg I University \\ Illkirch-graffenstaden, France
}

\author{
J. Baruthio, J.-P. Armspach \\ IPB, UMR 7004 CNRS-ULP \\ Strasbourg I University \\ Strasbourg, France
}

\author{
C. Maillot \\ Institut d'Anatomie Normale \\ Strasbourg I University \\ Strasbourg, France
}

\begin{abstract}
Magnetic resonance angiography (MRA) is currently used for cerebral flowing blood visualization. Many segmentation methods have been proposed for brain vessel segmentation, in order to help analyzing the huge data (generally more than $10^{7}$ voxels) provided by MRA acquisitions. Recently, a new family of segmentation algorithms, involving high level anatomical knowledge, has been studied. These new algorithms require a way to model and store this knowledge. An efficient and general approach to reach that goal consists in using atlases. In this paper a method is proposed to create vascular atlases of the brain, containing information useful for vessel segmentation purpose. This atlas creation process, designed for phase-contrast MRA (PC-MRA), is composed of four steps: segmentation, quantification, registration and data fusion. It uses a regiongrowing algorithm for vessel segmentation, a skeleton and vessel size determination algorithm, based on discrete geometry, for determination of quantitative properties, and a topology preserving non-rigid registration method to fuse the information. This method, which has been applied to a 18 PC-MRA database, enables to create vascular atlases containing information on brain vessels position, density, size and orientation. The generated atlases are essentially devoted to segmentation purpose but can also be used for anatomical description or pathology detection.
\end{abstract}

\section{Introduction}

Magnetic resonance angiography (MRA) is a family of non-invasive image acquisition processes providing 3D data of the flowing blood. One of these techniques, called phasecontrast MRA [1] (PC-MRA), is frequently used to study the cerebral vascular structures. Indeed, the availability of precise information about brain vascular networks is fundamental not only for planning and performing neurosurgi- cal procedures, but also for detecting pathologies such as aneurysms and stenoses. Many vessel segmentation methods $[2,3,4]$ have been proposed during the last 15 years (see [5] for an overview). Recently, a new family of segmentation algorithms [6] has been studied, involving high level anatomical knowledge. These algorithms are based on the hypothesis that a priori knowledge concerning the vessels and their relations to other brain structures can be used to guide classical segmentation tools. This kind of strategy requires an accurate way to model and store such anatomical information. A common way generally consists in using atlases. Nevertheless, since vessels are structures exhibiting a high variability, vascular atlas creation is a real challenge. We propose here a method to create such atlases (essentially devoted to vessel segmentation purpose) from a PC-MRA database. This method is composed of four successive steps: a region-growing vessel segmentation, a quantitative vessel analysis (determining vessels centerline and size), a non-rigid registration of the different images on a same reference, and the fusion of these registered images into a single one.

This paper is organized as follows. In Section 2, we review previous results concerning cerebral vascular atlases formation and use. In Section 3, we describe knowledge generally used by vessel segmentation algorithms and the high level anatomical knowledge that we propose to integrate in the atlas. Section 4 describes the different steps of the atlas generation algorithm. In Section 5, the method is applied on a 18 PC-MRA database, the resulting atlas is then described and analyzed. Some possible applications are then discussed in Section 6. Discussion and projects are finally presented in Section 7.

\section{Previous Work}

Atlases have already been designed for non vascular cerebral structure segmentation [7]. Nevertheless, the tor- 
tuosity and variability of brain vessels make the creation of vascular atlases a hard task. Despite these difficulties, two atlases have already been proposed.

In $[8,9]$, Chillet et al. and Cool et al. propose an atlas generation process. Vessels are extracted from an image database. An inverted distance map is then computed for each segmented image, providing a blurred vasculature. An arbitrary image is defined as the reference template. All the other ones are then mapped on this template, using affine registration. The mean and variance images finally obtained constitute the atlas.

In [10], the proposed atlas has been designed from a PCMRA magnitude image (similar to a classical MRI), using manual and semi-automated segmentation tools. Its goal was to propose a set of regions presenting homogeneous properties concerning vessel size, orientation and position relative to non vascular brain structures. This atlas was then used for segmentation purpose.

To the best of our knowledge, these two atlases constitute the very first attempts to model anatomical information concerning cerebral vascular structures. Despite their originality, both present drawbacks. The atlas proposed in $[8,9]$ is automatically generated by directly registering the segmented vessels. Thus, it does not take into account the anatomy of the neighboring cerebral structures. Moreover, the registration used is an affine transform, less reliable than non-rigid registration. Finally, the proposed atlas is not devoted to segmentation purpose. The atlas proposed and fully described in [10] is designed for segmentation purpose and takes into account information concerning the vessels in relation with other cerebral structures. Despite these advantages, it has not been automatically generated, and can contain imperfections. It also has to be noticed that it divides the brain into a finite set of vascular areas separated by fuzzy frontiers. This finite number of regions could be considered as a weakness. The method proposed hereafter has been designed to avoid all these drawbacks.

\section{Useful knowledge for segmentation}

Many methods have been proposed for vessel segmentation purpose [5], and more especially for brain vessel segmentation. Nevertheless, nearly all these methods use very little a priori knowledge. Indeed, they are generally based on three main hypotheses: vessels (i.e. flowing blood) correspond to the voxels of highest intensity in angiographic data; vessels are assumed to have a tubular shape; vascular networks are organized in a tree structure.

Although these information can be useful to guide segmentation algorithms, many other criteria could be taken into account, such as vessel size, orientation, and position according to non vascular cerebral structures. As an example, arteries located in the falx cerebri and vessels located
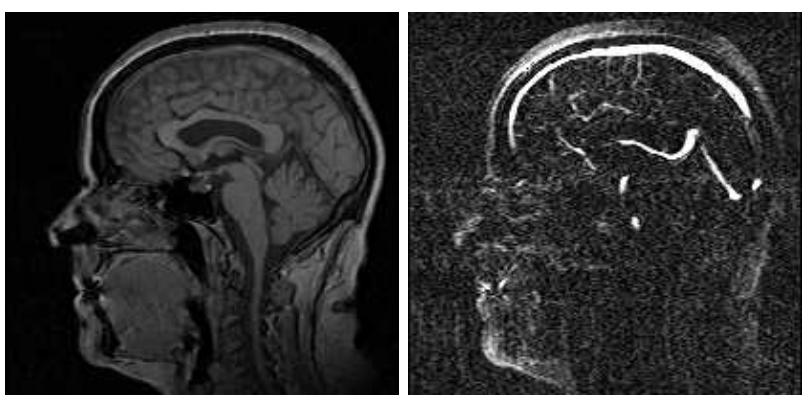

Figure 1. PC-MRA sagittal slices. Left: magnitude image slice. Right: phase image slice. Since images are generated during the same acquisition, they are perfectly superimposed.

in the neck have very different sizes and orientations, the first ones being small and oriented in a sagittal plane while the others are vertical and are much larger. The probability of detecting a vessel at a precise position, or the probability to find a vein or an artery can also be important for vessel segmentation and labeling. In the present approach, we propose to model information concerning vessel size and orientation but also the probability to find vascular structures at a given position. These pieces of knowledge are then stored in a cerebral vascular atlas. This atlas is generated using PC-MRA data (see Figure 1), enabling to take advantage of the bimodality of the acquired images during the generation process. Its main goal is to be used for MRA segmentation guidance. Nevertheless, it could also be used as a reference in order to detect abnormal or pathological vascular structures.

\section{Method}

\subsection{Definitions and notations}

A PC-MRA data is composed of two 3D images: a magnitude image, similar to a classical MRI, and a phase image, only containing moving structure signal (flowing blood, plus noise and artifacts). Sagittal slices of PC-MRA magnitude and phase images are illustrated in Figure 1. In the following, a PC-MRA data will be denoted by $p$ while a set of PC-MRA will be denoted by $P$. The magnitude image (resp. phase image) associated to $p$ will then be denoted by $p_{\text {mag }}$ (resp. $p_{p h a}$ ). Since phase and magnitude images of $p$ are 3D grey-level images, they can be defined as functions. Then, if $d_{x}, d_{y}, d_{z}$ are the dimensions of $p$, we have:

$$
\begin{array}{cc}
p_{\alpha}:\left[0, d_{x}-1\right] \times\left[0, d_{y}-1\right] \times\left[0, d_{z}-1\right] & \rightarrow \mathbb{N} \\
(x, y, z) & \mapsto v
\end{array},
$$

where $\alpha=\operatorname{mag}$ or $p h a$ and $v$ is the grey-level value of the image at the current point. For simplicity's sake, we will 

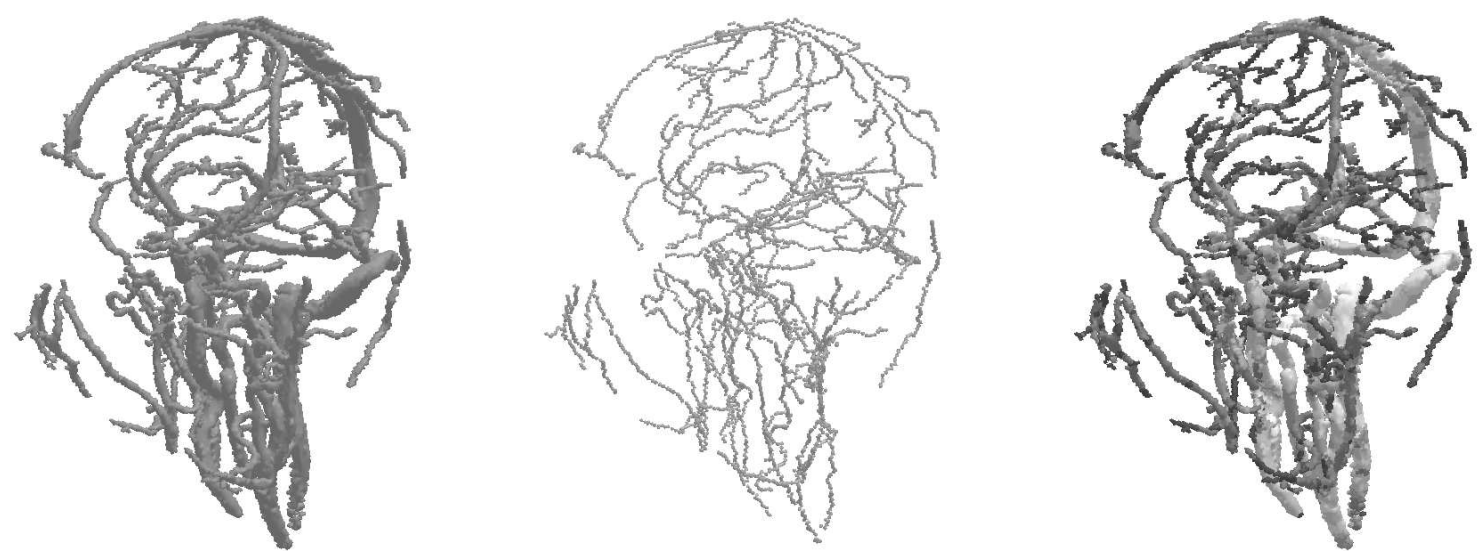

Figure 2. 3D visualization of the computed parameters for an image $p^{i}$ of the database $P$. From left to right: segmented vessels $\left(p_{s e g}^{i}\right)$, skeleton $\left(p_{s k e}^{i}\right)$, vessel diameter $\left(p_{t h i}^{i}\right)$.

consider that for a given set $P$, all $p \in P$ have the same dimensions $d_{x}, d_{y}, d_{z}$. The set $\left[0, d_{x}-1\right] \times\left[0, d_{y}-1\right] \times$ $\left[0, d_{z}-1\right]$ will be denoted by $I$ while an element $(x, y, z) \in$ $I$ will be denoted by $\mathbf{x}$ from now on.

\subsection{Input and output}

The method takes as input a PC-MRA database $P=$ $\left\{p^{i}\right\}_{i=0}^{n-1}$ of arbitrary size $n$ and a PC-MRA magnitude image $p_{m a g}^{r e f}$ used as a reference image for the registration step $\left(p_{m a g}^{r e f}\right.$ can be chosen in $P$ ). It provides as output a vascular atlas $\mathcal{A}$ defined by:

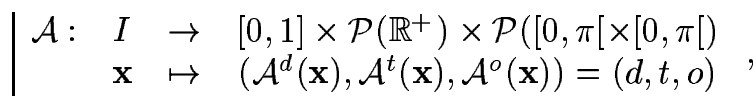

where $\mathcal{P}(X)=\{x \mid x \subseteq X\}$ stands for the set of all subsets of a set $X$, and $(d, t, o)$ represents the probability to find a vessel $(d)$, the possible diameters of a vessel $(t)$, and the set of its possible orientations $(o)$ at the current position $\mathbf{x}$.

\subsection{Algorithm}

Segmentation. The vessel segmentation is the first step of the process. It takes as input $P=\left\{p^{i}\right\}_{i=0}^{n-1}$ and computes the segmentation of $p^{i}$ for $i=0$ to $n-1$. The segmentation method, fully described in [10], is based on a region-growing algorithm using anatomical a priori knowledge and topological criteria. However, any vessel segmentation method providing a discrete binary image as result could be used. The segmentation of $p^{i}$ provides a 3D binary image $p_{s e g}^{i}$ containing the arterial and venous structures contained in $p^{i}$ :

$$
\mid \begin{array}{cccc}
p_{\text {seg }}^{i}: & I & \rightarrow & \{0,1\} \\
& \mathbf{x} & \mapsto & d
\end{array},
$$

where $d=1$ if a vessel is located at $\mathbf{x}$, and 0 otherwise. After the segmentation step, a new set $P_{\text {seg }}=\left\{p_{\text {seg }}^{i}\right\}_{i=0}^{n-1}$ of segmented images associated to $P$ is available. A segmented image example is illustrated in the left part of Figure 2.

Quantitative analysis. The set $P_{\text {seg }}$ can be used to compute the probability of finding a vascular structure at a given position. Nevertheless, the segmentation step does not provide any information on properties such as vessel diameter and orientation. In order to compute these parameters, every binary image $p_{\text {seg }}^{i}$ is processed by a diameter evaluation method proposed by Chillet et al., and fully described in [11]. This method is based on a skeletonization of the binary image. The skeleton, which contains information on vessel orientation, also enables to compute normal discrete planes used for vessel diameter evaluation. That step finally provides, for all $p^{i} \in P$ a 3D image $p_{t h i}^{i}$ :

$$
\mid \begin{array}{llll}
p_{t h i}^{i}: & I & \rightarrow & \mathbb{R}^{+} \\
& \mathbf{x} & \mapsto & t
\end{array},
$$

where $p_{t h i}^{i}(\mathbf{x})=t$ is the vessel diameter in the neighborhood of $\mathbf{x}$. It also provides a second 3D image $p_{s k e}^{i}$ :

$$
\begin{array}{|cccc}
p_{s k e}^{i}: & I & \rightarrow & {[0, \pi[\times[0, \pi[} \\
& \mathbf{x} & \mapsto & \left(p_{s k e, \theta}^{i}(\mathbf{x}), p_{s k e, \phi}^{i}(\mathbf{x})\right)=o
\end{array}
$$

where $p_{\text {ske }}^{i}(\mathbf{x})=o$ is the $3 \mathrm{D}$ orientation of the vessel in the neighborhood of $\mathbf{x}\left(p_{t h i}^{i}(\mathbf{x})\right.$ and $p_{s k e}^{i}(\mathbf{x})$ are defined if and only if $p_{\text {seg }}^{i}(\mathbf{x}) \neq 0$.). It has to be noticed that $\theta$ represents the angular position of the vessel by comparison to the vertical axis (where $\theta=0$ ), while $\phi$ is the angular position of the vessel by comparison to the sagittal axis (where $\phi=0)$. After this step, two new sets $P_{t h i}=\left\{p_{t h i}^{i}\right\}_{i=0}^{n-1}$ and $P_{s k e}=\left\{p_{s k e}^{i}\right\}_{i=0}^{n-1}$ of diameter and orientation images 
associated to $P$ are available. Examples of skeleton and diameter images are respectively illustrated in the middle and right parts of Figure 2.

Non-rigid registration. In order to correctly combine the information of every image of the database, it is necessary that the fused values correspond to the same position in the brain or the head. Indeed, even though the principal parts of the human brain are quite similar from one person to another, their size and proportions can be different. It is then fundamental to find, for every processed image, a correct $3 \mathrm{D}$ deformation field enabling to map them on a same reference. This is what is done by the non-rigid registration. The registration algorithm used here is the one proposed by Noblet et al. in [12]. It has been chosen for its ability to preserve the topology of the registered anatomical structures.

For all $p_{m a g}^{i} \in P$, the PC-MRA magnitude image $p_{m a g}^{i}$ is registered on the reference magnitude image $p_{m a g}^{r e f}$. This registration provides a $3 \mathrm{D}$ deformation field $D^{i}$. Then for any $\mathbf{x} \in I, p^{i}(\mathbf{x})$ is assumed to be equal to $p_{m a g}^{r e f}\left(D^{i}(\mathbf{x})\right)$, from an anatomical point of view.

Data fusion. The last step consists in fusing the pieces of knowledge of each image of the database. The first part of the atlas:

$$
\mid \begin{array}{cccc}
\mathcal{A}^{d}: & I & \rightarrow & {[0,1]} \\
& \mathbf{x} & \mapsto & d
\end{array},
$$

is defined such as:

$$
\forall \mathbf{x} \in I, \mathcal{A}^{d}(\mathbf{x})=\frac{1}{n} \sum_{i=0}^{n-1} p_{s e g}^{i}\left(\left(D^{i}\right)^{-1}(\mathbf{x})\right) .
$$

By definition, $\mathcal{A}^{d}(\mathbf{x}) \in[0,1]$ and provides the probability to find a vascular structure at the current position $\mathrm{x}$ of $p_{m a g}^{r e f}$.

The second part of the atlas:

$$
\begin{array}{cccc}
\mathcal{A}^{t}: & I & \rightarrow & \mathcal{P}\left(\mathbb{R}^{+}\right) \\
& \mathbf{x} & \mapsto & t
\end{array},
$$

is defined such as:

$\forall \mathbf{x} \in I, \mathcal{A}^{t}(\mathbf{x})=\left[\max \left\{0, a(\mathbf{x})-\sigma_{a}(\mathbf{x})\right\}, a(\mathbf{x})+\sigma_{a}(\mathbf{x})\right]$,

where:

$$
a(\mathbf{x})=\left\{\begin{array}{lr}
0 & \text { if } N(\mathbf{x})=\emptyset \\
\frac{1}{\# N(\mathbf{x})} \sum_{i \in N(\mathbf{x})} p_{t h i}^{i}\left(\left(D^{i}\right)^{-1}(\mathbf{x})\right) \\
\text { otherwise }
\end{array}\right.
$$

with:

$$
N(\mathbf{x})=\left\{i \in[0, n-1] \mid p_{\text {seg }}^{i}\left(\left(D^{i}\right)^{-1}(\mathbf{x})\right)=1\right\},
$$

$\# X$ standing for the cardinal of a set $X$, and $\sigma_{a}(\mathbf{x})$ standing for the standard deviation associated to $a(\mathbf{x})$. This definition, using the standard deviation and the average value of the vessel diameters, enables to take into account their variability between the different cases.

The last part of the atlas:

$$
\mid \begin{array}{cccc}
\mathcal{A}^{o}: & I & \rightarrow & \mathcal{P}([0, \pi[\times[0, \pi[) \\
& \mathbf{x} & \mapsto & o
\end{array}
$$

is defined such as:

$$
\forall \mathbf{x} \in I, \mathcal{A}^{o}(\mathbf{x})=\Theta(\mathbf{x}) \times \Phi(\mathbf{x})
$$

where:

$$
\begin{gathered}
\Theta(\mathbf{x})=\left\{\begin{array}{lr}
\emptyset & \text { if } N(\mathbf{x})=\emptyset \\
{[0, \pi[} & \text { if } N(\mathbf{x}) \neq \emptyset \text { and } \sigma_{\theta}(\mathbf{x}) \geq \pi / 4 \\
{\left[\theta(\mathbf{x})-\sigma_{\theta}(\mathbf{x}), \theta(\mathbf{x})+\sigma_{\theta}(\mathbf{x})\right](\bmod \pi)} & \text { otherwise }
\end{array}\right. \\
\Phi(\mathbf{x})=\left\{\begin{array}{lr}
\emptyset & \text { if } N(\mathbf{x})=\emptyset \\
{[0, \pi[} & \text { if } N(\mathbf{x}) \neq \emptyset \text { and } \sigma_{\phi}(\mathbf{x}) \geq \pi / 4 \\
{\left[\phi(\mathbf{x})-\sigma_{\phi}(\mathbf{x}), \phi(\mathbf{x})+\sigma_{\phi}(\mathbf{x})\right](\bmod \pi)}
\end{array}\right. \\
\end{gathered}
$$

with:

$$
\begin{aligned}
& \theta(\mathbf{x})=\sum_{i \in N(\mathbf{x})} \frac{1}{\# N(\mathbf{x})} p_{s k e, \theta}^{i}\left(\left(D^{i}\right)^{-1}(\mathbf{x})\right) \\
& \phi(\mathbf{x})=\sum_{i \in N(\mathbf{x})} \frac{1}{\# N(\mathbf{x})} p_{s k e, \phi}^{i}\left(\left(D^{i}\right)^{-1}(\mathbf{x})\right),
\end{aligned}
$$

and $\sigma_{\theta}(\mathbf{x})$ (resp. $\sigma_{\phi}(\mathbf{x})$ ) standing for the standard deviation associated to $\theta(\mathbf{x})$ (resp. $\phi(\mathbf{x})$ ). It has been chosen to set $\Theta(\mathbf{x})$ (resp. $\Phi(\mathbf{x})$ ) to $[0, \pi[$, i.e. its maximum size, if the standard deviation was higher than $\pi / 4$. Indeed, if this case occurs, one can consider that there is no significant vessel orientation at the position $\mathbf{x}$. These three parts, $\mathcal{A}^{d}, \mathcal{A}^{t}$, and $\mathcal{A}^{o}$ finally provide the whole atlas $\mathcal{A}$.

\subsection{Implementation}

The proposed method has been implemented on the Medimax $^{1}$ software platform and use the $\mathrm{ImLib} \mathrm{D}^{2}$ open source C++ library [13]. The computer used to run the method was composed of a $3 \mathrm{GHz}$ Pentium IV processor with 2 GB of memory. The successive steps of the algorithms respectively required 45,6 , and 40 minutes for each image of the database. The data fusion required 15 minutes for a 18 image database. Since the non-rigid registration step is independent from the segmentation and quantitative analysis steps, it could be possible to simultaneously carry out these steps on different computers, thus reducing the computation time.

\footnotetext{
${ }^{1}$ Available at http://www-ipb.u-strasbg.fr.

${ }^{2}$ Available at http://imlib3d. sourceforge. net.
} 
It has to be noticed that the final atlas $\mathcal{A}$ can be modeled by the following function:

$$
\mid \begin{array}{cccc}
\mathcal{A}^{\prime}: & I & \rightarrow & \mathbb{R}^{7} \\
& \mathbf{x} & \mapsto & \left(d, a, \sigma_{a}, \theta, \sigma_{\theta}, \phi, \sigma_{\phi}\right)(\mathbf{x})
\end{array}
$$

which enables to store it in a simple way and to easily recover any useful information it contains.

\section{Atlas generation}

\subsection{Imaging and subjects}

A 18 PC-MRA database has been used to create the atlas proposed in this paper. The MRA exams were performed on a 1 Tesla whole-body scanner (Gyroscan NT/INTERA $1.0 \mathrm{~T}$ from Philips, gradient slope $75 \mathrm{~T} / \mathrm{m} / \mathrm{s}$ ). The flow encoding sequence called T1FFE/PCA uses a TR of $10 \mathrm{~ms}$ and a TE of $6.4 \mathrm{~ms}$. The pool of patients was composed of males and females aged from 21 to 80 without any cerebral pathologies. The acquired images of dimensions varying from $256^{2} \times 150$ to $256^{2} \times 180$ voxels, were made of non-isotropic voxels. The images have then been processed to provide cubic voxels. Indeed, the determination of vessel criteria such as diameter or centreline is more accurate when performed on isotropic data.

\subsection{Atlas description}

The obtained atlas has been computed using a reference PC-MRA magnitude image previously called $p_{m a g}^{r e f}$. By definition, the atlas presents the same properties as $p_{m a g}^{r e f}$. It is composed of $256 \times 229 \times 160$ voxels. The voxels are cubes of $1.13 \mathrm{~mm}$ edges.

Vascular density. The vascular density represents the probability to find a vessel at a given position $\left(\mathcal{A}^{d}\right)$. A spatial representation of this probability field is illustrated in the left part of Figure 3. One can clearly observe invariant structures such as many veins and sinuses (superior sagittal sinus, straight sinus, transverse and sigmoid sinuses, jugular veins) but also arterial structures (carotid and pericallosal arteries). Other vessels appear as "vascular clouds", such as many arteries located in the medial part of the brain. This phenomenon can be explained by their high position variability.

A more quantitative representation of these probabilities is proposed in Table 1. It has to be noticed that the vessels are localized in a very small part of the whole image. Indeed, less than $4 \%$ of the atlas is assumed to contain vessels sufficiently large to be visualized in MRA data. This information can be of precious use for computation time reduction in segmentation processes.

\begin{tabular}{|c|r|r|}
\hline Probability & \multicolumn{1}{|c|}{ \# voxels } & \multicolumn{1}{|c|}{ Ratio } \\
\hline$p=0$ & 9028698 & $96.18 \%$ \\
$0<p \leq 0.1$ & 209718 & $2.23 \%$ \\
$0.1<p \leq 0.2$ & 87390 & $0.93 \%$ \\
$0.2<p \leq 0.3$ & 27031 & $0.29 \%$ \\
$0.3<p \leq 0.4$ & 13192 & $0.14 \%$ \\
$0.4<p \leq 0.5$ & 7092 & $0.08 \%$ \\
$0.5<p$ & 6719 & $0.07 \%$ \\
\hline$p \neq 0$ & 351142 & $3.74 \%$ \\
\hline
\end{tabular}

Table 1. Distribution of the probability $(p)$ to find a vascular structure. First column: values of $p$; second and third columns: number and ratio of voxels $\mathbf{x}$ such as $\mathcal{A}^{d}(\mathbf{x})=p$.

Vessel diameter. The generated atlas also provides information concerning diameter of the different vessels. A MIP of the diameter image of the atlas is proposed in the middle part of Figure 3. The biggest vessels are localized in the neck and in the venous parts while the positions corresponding to arterial structures present smaller ones. The distribution of vessel diameters is summarized in Table 2. It has to be noticed that very few diameters lower than 1 $\mathrm{mm}$ are observed. This is explained by the limitations of the acquisition process (MRA) which still does not enable to obtain submillimetric data.

\begin{tabular}{|c|r|r|}
\hline Diameter $(\mathrm{mm})$ & \# voxels & \multicolumn{1}{|c|}{ Ratio } \\
\hline $0<t \leq 1.0$ & 5066 & $1.44 \%$ \\
$1.0<t \leq 2.0$ & 80282 & $22.86 \%$ \\
$2.0<t \leq 3.0$ & 111372 & $31.72 \%$ \\
$3.0<t \leq 4.0$ & 80139 & $22.82 \%$ \\
$4.0<t \leq 5.0$ & 56547 & $16.10 \%$ \\
$5.0<t \leq 6.0$ & 14411 & $4.10 \%$ \\
$6.0<t$ & 3121 & $0.89 \%$ \\
\hline
\end{tabular}

Table 2. Distribution of the average diameter of the vessels in the vascular part of the image (part presenting non zero values for $\mathcal{A}^{d}$ ). First column: average diameter $(t)$; second and third columns: number and ratio of voxels $\mathbf{x}$ such as $a(\mathbf{x})=t$.

Vessel orientation. The atlas finally provides information concerning the orientation of the cerebral vascular structures. A 3D visualization of the orientations for the left part of the atlas is illustrated in the right part of Figure 3. The distribution of the orientations is summarized in Table 3.

One can observe that most of the voxels presenting a defined $\theta$ orientation have a quite horizontal one $\theta \in$ $[\pi / 3,2 \pi / 3])$. These voxels belong to cerebral parts of the atlas containing venous structures which are often oriented in a nearly horizontal plane (superior sagittal sinus, straight sinus). Most of these structures also present a $\phi$ orientation close to the sagittal plane which explains the high ratio of voxels such as $\phi \in[\pi / 3,2 \pi / 3]$. However, a higher amount of voxels presenting a vertical orientation $(\theta \in[0, \pi / 6] \cup[5 \pi / 6, \pi])$ could have been expected. This can be explained by the fact that many vessels presenting 

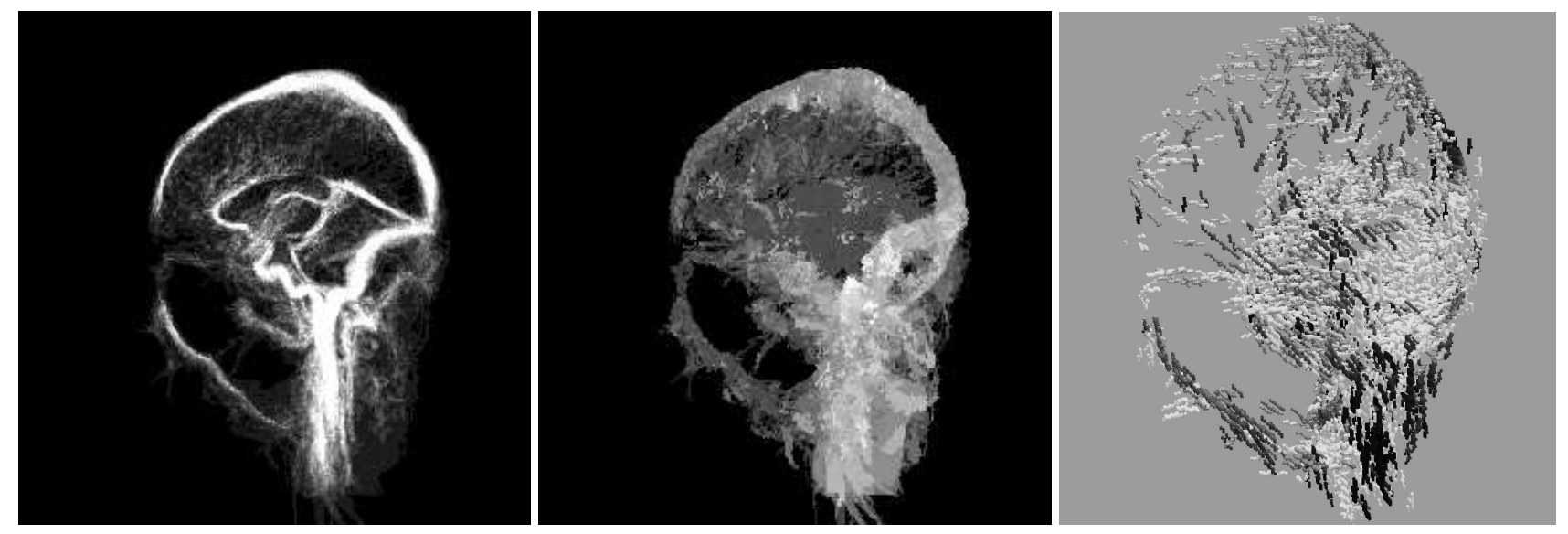

Figure 3. Visualization of elements of the processed atlas. Left: probability to find a vessel $\left(\mathcal{A}^{d}\right)$, visualized as a maximum intensity projection of the sagittal slices; the brighter the region, the higher the probability to find a vessel. Middle: average vessel diameters (part $a$ of $\mathcal{A}^{t}$ ), visualized as a maximum intensity projection of the sagittal slices; the brighter the region, the larger the vessels. Right: 3D visualization of a part of the orientation image (lines oriented according to $\theta$ and $\phi$ ); the grey level linearly depends on the $\theta$ value.

a vertical orientation are located in the neck (carotid arteries, jugular veins). Indeed, the non rigid registration used in this method, which is essentially devoted to brain registration, provides satisfactory results for cerebral structures, but less accurate ones for those located in the neck. Then the standard deviation values generally obtained in this area were not sufficiently low to define the orientation.

More generally, the amount of voxels presenting defined orientation could be significantly increased by no longer considering the absolute orientation of the vessels but their relative orientation according to adjacent non vascular structures. As an example, the superior sagittal sinus relative orientation according to the surface of the skull is quite invariant. The same property can be observed for many vascular and non vascular structures (straight sinus and superior frontier of the cerebellum, brain superficial veins and cortex). In order to be modeled, such properties would require a fusion between an atlas as the one presented here and non vascular brain atlases.

\begin{tabular}{|l|r|r|r|r|}
\hline \multirow{2}{*}{$\begin{array}{l}\text { Orientation } \\
\text { (rad.) }\end{array}$} & \multicolumn{2}{|c|}{$\theta$} & \multicolumn{2}{c|}{$\phi$} \\
\cline { 2 - 5 } \# voxels & \multicolumn{1}{|c|}{ Ratio } & \# voxels & \multicolumn{1}{c|}{ Ratio } \\
\hline Undefined & 253978 & $72.33 \%$ & 261496 & $74.48 \%$ \\
{$[0, \pi / 6[$} & 3540 & $1.01 \%$ & 7298 & $2.08 \%$ \\
{$[\pi / 6, \pi / 3[$} & 14931 & $4.25 \%$ & 24873 & $7.08 \%$ \\
{$[\pi / 3, \pi / 2[$} & 31174 & $8.88 \%$ & 21669 & $6.17 \%$ \\
{$[\pi / 2,2 \pi / 3[$} & 27679 & $7.88 \%$ & 16898 & $4.81 \%$ \\
{$[2 \pi / 3,5 \pi / 6[$} & 13597 & $3.87 \%$ & 14685 & $4.18 \%$ \\
{$[5 \pi / 6, \pi[$} & 6243 & $1.78 \%$ & 4223 & $1.20 \%$ \\
\hline
\end{tabular}

Table 3. Distribution of the orientation of the vessels in the vascular part of the image (part presenting non zero values for $\mathcal{A}^{d}$ ). First column: values of $\theta$ or $\phi$; second and third (resp. fourth and fifth) columns: number and ratio of voxels presenting the current orientation according to $\theta$ (resp. $\phi$ ).

\section{Possible use of atlases}

\subsection{Knowledge-based segmentation and filtering}

The atlases that can be generated by the proposed method are essentially devoted to segmentation purpose. Indeed, reliable a priori information concerning vessel position, diameter, and orientation can be very useful for guidance of several kinds of methods.

Concerning methods based on filtering or application of mathematical morphology operators, this knowledge can be used to reduce the computation time without altering the accuracy of the result. Indeed, these methods are generally designed to filter each voxel of the image with a set of operators presenting different parameters. A priori knowledge can then be used to decide which areas of the whole image need to be processed or not, and to choose specific subsets among the whole set of possible parameters. Such an approach is proposed in [6], for brain vessel segmentation based on grey-level hit-or-miss transform.

A priori information can also be used in order to improve the efficiency of deformable models algorithms. This kind of methods generally requires an accurate initialization to provide correct results (an approximate initialization can lead to an erroneous segmentation). Information on position, size and orientation of the vessels could then allow to efficiently and automatically generate and position an initial model shape close from the structures to be segmented. These parameters could also be used to guide the model evolution, by integrating them in the energy function to minimize.

Other applications of vascular atlases could be cited, 
such as automatic initialization of vessel tracking algorithms, or guidance of region growing methods.

\subsection{Computer-aided diagnosis and anatomical comparison}

Assuming that the method is applied on a set of people without any cerebral pathologies, the resulting atlas could be used as a reference for analyzing MRA of non-healthy patients and detecting pathologies. The most common vascular pathologies are aneurysms (broadening of vessels) and stenoses (narrowing of vessels), corresponding to vessel diameter abnormal modifications. Since an atlas provides an estimation of the expected diameter for each main vessel of the brain and the whole head, it could be a useful reference to help the clinicians to automatically detect possibly pathological structures. Such an atlas could also be used for anatomical comparison, in order to determine the variability of the different vascular structures of the brain. This could lead to the creation of reliable and exhaustive descriptions of these structures, taking into account all their different configurations.

\section{Conclusion}

This paper has presented a novel method for automatic generation of vascular atlases from any arbitrary PC-MRA database. This method is composed of four steps involving vessel segmentation, quantification, non-rigid registration and data fusion. It enables to compute, in an automatic fashion, atlases modeling high level anatomical information (position, size, orientation) on brain vessels. Such atlases are then assumed to be more reliable and to contain more useful information than those previously proposed. Although these atlases can be used for computer-aided diagnosis, their main goal remains smart guidance of segmentation tools, such as those based on mathematical morphology operators. Further work will now consist in modeling more knowledge as vessel shape, or vein/artery discrimination for labeling purpose, but also to obtain better results concerning vessel orientation. Moreover the fusion of vascular and non vascular atlases constitutes a promising research field which could lead to quite accurate vessel description tools.

\section{Acknowledgments}

The authors thank the EPML IRMC ${ }^{3}$ (Équipe-Projet Multi-Laboratoires Imagerie et Robotique Médicale et Chirurgicale, EPML \#9 CNRS-STIC) for its financial support.

\footnotetext{
${ }^{3}$ http://irmc.u-strasbg.fr
}

\section{References}

[1] C.L. Dumoulin, S.P. Souza, M.F. Walker, W. Wagle, "Threedimensional phase contrast angiography," Magnetic Resonance in Medicine, Vol. 9, pp. 139-149, 1989.

[2] N. Flasque, M. Desvignes, J.M. Constans, M. Revenu, "Acquisition, segmentation and tracking of the cerebral vascular tree on 3D magnetic resonance angiography images," Medical Image Analysis, Vol. 5, pp. 173-183, 2001.

[3] S. Kobashi, N. Kamiura Y. Hata, F. Miyawaki, "Volumequantization-based neural network approach to 3D MR angiography image segmentation," Image and Vision Computing, Vol. 19, pp. 185-193, 2001

[4] A.C.S. Chung, J.A. Noble, "Statistical 3D vessel segmentation using a rician distribution," Proc. MICCAI 99, in LNCS, Vol. 1679, pp. 82-89, 1999.

[5] S. Suri, K. Liu, L. Reden, S. Laxminarayan, "A review on MR vascular image processing: Skeleton versus nonskeleton approaches: Part II,' IEEE Transactions on Information Technology in Biomedicine, Vol. 6, pp. 338-350, 2002.

[6] N. Passat, C. Ronse, J. Baruthio, J.-P. Armspach, "Automatic parameterization of grey-level hit-or-miss operators for brain vessel segmentation," Proc. IEEE ICASSP 2005, Vol II, pp. 737-740.

[7] O. Musse, F. Heitz, J.-P. Armspach, "Fast deformable matching of 3D images over multiscale nested subspaces. Application to atlas-based MRI segmentation," Pattern Recognition, Vol. 36, pp. 1881-1899, 2003.

[8] D. Chillet, J. Jomier, D. Cool, S. Aylward "Vascular atlas formation using a vessel-to-image affine registration method," Proc. MICCAI 2003, in LNCS, Vol. 2879, pp. 335-342, 2003.

[9] D. Cool, D. Chillet, J. Kim, S. Aylward, "Tissue-based affine registration of brain images to form a vascular density atlas," Proc. MICCAI 2003, in LNCS, Vol. 2879, pp. 9-15, 2003.

[10] N. Passat, C. Ronse, J. Baruthio, J.-P. Armspach, C. Maillot, C. Jahn, "Region growing segmentation of brain vessels: An atlas-based automatic approach," Journal of Magnetic Resonance Imaging, to appear.

[11] D. Chillet, N. Passat, M.-A. Jacob-Da Col, J. Baruthio, "Thickness estimation algorithm for discrete tree-like tubular objects," Submitted.

[12] V. Noblet, C. Heinrich, F. Heitz, J.-P. Armspach, "A topology preserving non-rigid registration method using a symmetric similarity function - Application to 3-D brain images," Proc. ECCV 2004, in LNCS, Vol. 3023, pp. 546-557, 2004.

[13] M. Bosc, T. Vik, J.-P. Armspach, F. Heitz, "ImLib3D: An efficient, open source, medical image processing framework in C++," Proc. MICCAI 2003, in LNCS, Vol. 2879, pp. 981982, 2003. 